BULLETIN (New Series) OF THE

AMERICAN MATHEMATICAL SOCIETY

Volume 40, Number 2, Pages 179-201

S 0273-0979(03)00976-5

Article electronically published on February 12, 2003

\title{
THE INTERPLAY BETWEEN ANALYSIS AND TOPOLOGY IN SOME NONLINEAR PDE PROBLEMS
}

\author{
HAIM BREZIS
}

\section{From the LAPLACE EQUATION TO HARMONIC MAPS: A HISTORICAL PERSPECTIVE}

Let us recall the formulation of the standard Dirichlet problem for the Laplace operator. Let $\Omega \subset \mathbb{R}^{n}$ be a smooth bounded domain and let $\varphi: \partial \Omega \rightarrow \mathbb{R}$ be a given function. The problem is to find a (smooth) function $u: \Omega \rightarrow \mathbb{R}$ satisfying

$$
\begin{array}{cc}
\Delta u=\sum_{i=1}^{n} \frac{\partial^{2} u}{\partial x_{i}^{2}}=0 & \text { in } \Omega, \\
u=\varphi & \text { on } \partial \Omega .
\end{array}
$$

In a fundamental paper from 1890, H. Poincaré [49] (see also the lecture notes from a course of H. Poincaré [50] at the Sorbonne) gave the first complete proof of existence and uniqueness of a solution for problem $(0.1)-(0.2)$ when $n=2$ and $n=3$ :

Theorem 1 (H. Poincaré). Given any $\varphi \in C^{0}(\partial \Omega)$, there is a unique classical solution $u \in C^{\infty}(\Omega) \cap C^{0}(\bar{\Omega})$ of $(0.1)-(0.2)$.

The "balayage" method introduced by H. Poincaré in his proof of Theorem 1 relies heavily on tools of Potential theory: maximum principle, Harnack's inequality, explicit representation formulas for the Dirichlet problem in a ball (Poisson integral), etc.

In 1900, D. Hilbert [39], in a celebrated address, followed by a (slightly) more detailed paper in 1904, announced that he had solved the Dirichlet problem (0.1) (0.2) via the Dirichlet principle which had been discovered by G. Green in 1833, with later contributions by C. F. Gauss (1837), W. Thomson (=Lord Kelvin) (1847) and G. Riemann (1853). Dirichlet's principle asserts that any solution $u$ of (0.1) $(0.2)$ is a minimizer of the Dirichlet integral

$$
E(v)=\int_{\Omega}|\nabla v|^{2}=\sum_{i=1}^{n} \int_{\Omega}\left(\frac{\partial v}{\partial x_{i}}\right)^{2}
$$

Received by the editors October 23, 2002.

2000 Mathematics Subject Classification. Primary 35A15, 35A20, 35J50, 35J65, 35Qxx, $46 \mathrm{Txx}, 47 \mathrm{Hxx}, 47 \mathrm{Jxx}, 55 \mathrm{Pxx}, 58 \mathrm{E} 15$.

This text is an expanded version of the invited address at the AMS Meeting "Mathematical Challenges of the 21st Century", UCLA (2000). 
in the class of function $v: \Omega \rightarrow \mathbb{R}$ satisfying the boundary condition $v=\varphi$ on $\partial \Omega$. The existence of a minimizer for $E$ had been taken for granted until 1870, when $K$. Weierstrass pointed out that a rigorous proof was lacking. It is easy to see that any (classical) solution of $(0.1)-(0.2)$ is a minimizer for $E$. The touchy points are the following:

a) Prove directly, without invoking equations $(0.1)-(0.2)$, that $E$ admits a minimizer.

b) Prove that the minimizer (which belongs to the class of functions having finite energy) is smooth and satisfies (0.1) - (0.2).

The announcement of Hilbert turned out to be a little premature. Instead, it became a program which stimulated many people during the period 1900-1940: B. Levi, H. Lebesgue, L. Tonelli, R. Courant, S. L. Sobolev and many others.

In 1940, H. Weyl [60] completed Hilbert's program. By 1940 the Calculus of Variations had been placed on firm ground, and it provided a very fruitful link between PDE and Functional Analysis; for further discussions we refer the reader to the expository paper of H. Brezis and F. Browder [15].

Starting in the 1940's a number of mathematicians considered similar questions for systems. Namely, the unknown $u: \Omega \rightarrow \mathbb{R}$ becomes a vector-valued function $u$ : $\Omega \rightarrow \mathbb{R}^{k}$ or more generally $u: \Omega \rightarrow N$ where $N\left(=N^{k}\right)$ is a $k$-dimensional manifold (usually without boundary). The domain $\Omega \subset \mathbb{R}^{n}$ could also be replaced by a manifold $M\left(=M^{n}\right)$ (with or without boundary). The most natural generalization of the Dirichlet principle to this setting is the problem of harmonic maps. The energy of a map $u: M \rightarrow N$ is defined by

$$
E(u)=\int_{M} e(u) d V
$$

where the energy density is defined in local coordinates by

$$
e(u)=\sum_{i, j, \alpha, \beta} g_{i j}(u) \gamma^{\alpha \beta}(x) \frac{\partial u^{i}}{\partial x^{\alpha}} \frac{\partial u^{j}}{\partial x^{\beta}}
$$

and $\gamma^{\alpha, \beta}, g_{i j}$ are the metric tensors on $M$ and $N$. The corresponding Euler-Lagrange equation associated with the minimization of $E$ subject to the constraint $u: M \rightarrow$ $N$ is a system of nonlinear PDE's:

$$
-\Delta u=\Gamma(u)(\nabla u, \nabla u) \text { in } M,
$$

where $\Delta$ is the Laplace-Beltrami operator on $M$ and $\Gamma$ is a quadratic expression in $\nabla u$ related to the Christoffel symbols on $N$. The solutions of the equation (0.3), i.e., the critical points of $E$, are called the harmonic maps. When $M$ has a boundary $\partial M$, the PDE (0.3) is usually coupled with a Dirichlet boundary condition

$$
u=\varphi \quad \text { on } \partial M \text {. }
$$

Basic questions (existence, regularity, etc.) for systems of nonlinear PDE's, and in particular harmonic maps, became the subject of intensive investigations after the middle of the 20th century. Here are some names of leading contributors for the period 1940 to the early 80's:

- starting in the 40's: C. Morrey;

- starting in the 60's: E. DeGiorgi, E. Giusti, M. Miranda, F. Almgren, H. Federer, J. Eells, J. H. Sampson, etc.; 
- starting in the 70's: M. Giaquinta, R. Hamilton, S. Hildebrandt, R. Schoen, S. T. Yau, W. Jäger, etc.;

- starting in the early 80's: K. Uhlenbeck, L. Simon, J. Jost, L. Lemaire, B. White, etc.

The interested reader will find more information in the following books and review articles: C. Morrey [48], H. Federer 27], R. Hamilton 32, J. Eells and L. Lemaire 22, 23], S. Hildebrandt 40, M. Giaquinta 28], J. Jost 42, E. Giusti [31], R. Schoen and S. T. Yau [58.

Until the late 70's, the main motivation came from problems arising in Differential Geometry and some of the tools came from Geometric Measure theory. Starting in the mid 80's two new trends became highly visible:

On the one hand, the number of papers dealing with harmonic maps has increased enormously, giving an impression of chaotic growth. On the other hand, motivations coming from physics brought a refreshing wind of new problems and suggested new tools.

Here are a few topics in physics having connections with harmonic maps and some of the mathematicians involved in their study:

A) Yang-Mills equations and gauge theories (M. Atiyah, S. K. Donaldson, C. Taubes, K. Uhlenbeck, E. Witten, N. Seiberg, etc.)

B) Liquid crystals (J. Ericksen, D. Kinderlehrer, R. Hardt, F. H. Lin, H. Brezis, J.-M. Coron, E. Lieb, etc.)

C) Superfluids, superconductors, Ginzburg-Landau models (F. Bethuel, H. Brezis, F. Hélein, R. Hardt, F. H. Lin, T. Rivière, etc.)

D) Skyrmions (M. Esteban, E. Lieb, etc.)

Before getting into details, I would like to stress two major differences between the standard Dirichlet problem (0.1) - (0.2) and the theory of harmonic maps.

1) In the standard Dirichlet problem harmonic functions are smooth $\left(C^{\infty}\right)$ in $\Omega$. By contrast, harmonic maps have singularities. In fact, they often correspond to visible physical observations: point or line "defects", "vortices", etc. Sometimes singularities occur because of topological obstructions: for example some (smooth) topologically nontrivial boundary conditions on $\partial \Omega$ do not admit smooth extensions inside $\Omega$ (see the discussion in Section 2 and Section 4). Sometimes singularities appear even in the absence of topological obstructions simply because solutions with singularities have lower energy (seems paradoxical, but see Section 1.2). Here, the key words are partial regularity, i.e., the singular set of (some) harmonic maps is often "small" (it consists of isolated points, lines, etc.).

2) The fact that one deals with maps from the manifold $M$ into the manifold $N$ makes the underlying function spaces much richer from the point of view of Topology. Therefore one may hope to find multiple solutions - ideally (at least) one in each homotopy class. On the other hand, the standard homotopy classes of $C^{0}(M, N)$ need not be the relevant tool when dealing with singular solutions. The natural function space is the Sobolev space $H^{1}(M, N)$ and its connected component may be quite different from the ones of $C^{0}(M, N)$. For example if $M=N=S^{3}$, the space $H^{1}\left(S^{3}, S^{3}\right)$ is path-connected while $C^{0}\left(S^{3}, S^{3}\right)$ admits connected components (classified by their degree). It is necessary to revisit Homotopy theory in the framework of Sobolev spaces, and this is a whole new field under investigation; see Section 4. 
In this "labyrinth" of results I propose to concentrate on three very special cases. Each of them displays different features. Despite their simplicity these model cases illuminate general phenomena.

The plan is the following:

0. From the Laplace equation to harmonic maps: a historical perspective

1. Model Case I : $M=B^{3}$ and $N=S^{2}$

2. Model Case II : $M=B^{2}$ and $N=S^{2}$

3. Model Case III : $M=B^{2}$ (or $B^{3}$ ) and $N=S^{1}$

4. Homotopy theory in the framework of Sobolev spaces

Here $B^{k}$ denotes the unit ball in $\mathbb{R}^{k}$.

$$
\text { 1. Model Case I: } M=B^{3} \text { And } N=S^{2}
$$

In this section we take $\Omega=M=B^{3}$, the unit ball in $\mathbb{R}^{3}$, and $N=S^{2}$, the unit sphere in $\mathbb{R}^{3}$. This is a simplified model for some problems occurring in the theory of nematic liquid crystals (see e.g. the expository presentations in J. Ericksen [24], J. Ericksen and D. Kinderlehrer [25, M. Kléman [46] and the paper of R. Hardt, D. Kinderlehrer and F. H. Lin 34.)

1.1. The Dirichlet minimization problem. Given a smooth boundary condition $\varphi: \partial \Omega=S^{2} \rightarrow S^{2}$, we investigate the minimization problem

$$
\operatorname{Min}_{\substack{u: \Omega \rightarrow S^{2} \\ u=\varphi \text { on } \partial \Omega}} E(u), \text { where } E(u)=\int_{\Omega}|\nabla u|^{2} .
$$

The natural functional space is

$$
H_{\varphi}^{1}\left(\Omega, S^{2}\right)=\left\{u \in L^{2}\left(\Omega, S^{2}\right) ; \nabla u \in L^{2} \text { and } u=\varphi \text { on } \partial \Omega\right\} .
$$

It is easy to see that $H_{\varphi}^{1}$ is never empty; for example

$$
u(x)=\varphi(x /|x|)
$$

belongs to $H^{1}$ because in 3 -d

$$
\int_{\Omega}\left|\nabla\left(\frac{x}{|x|}\right)\right|^{2}<\infty
$$

Note that, by contrast,

$$
C_{\varphi}^{0}\left(\bar{\Omega}, S^{2}\right) \neq \phi \Longleftrightarrow \operatorname{deg}(\varphi)=0 .
$$

In particular, if $\operatorname{deg}(\varphi) \neq 0$, every $u \in H_{\varphi}^{1}\left(\Omega, S^{2}\right)$ must have at least one singularity. This is a typical situation where a topological condition forces the creation of singularities.

It is easy to see that the minimum in (1.1) is achieved and satisfies the harmonic map equation (0.3), which, in this special case, takes the form

$$
-\Delta u=u|\nabla u|^{2} \text { in } \Omega \text {. }
$$

Equation (1.2) is a coupled system:

$$
-\Delta u_{i}=u_{i}|\nabla u|^{2} \quad i=1,2,3
$$

where $|\nabla u|^{2}=\sum_{i, j}\left(\frac{\partial u_{i}}{\partial x_{j}}\right)^{2}$. A weak solution $u \in H_{\varphi}^{1}\left(\Omega, S^{2}\right)$ of (1.2) is called a weakly harmonic map. 
We now turn to the question of regularity. An important result is

Theorem 2 (R. Schoen and K. Uhlenbeck [55, [56, [57]). Assume u is a minimizer of $E$ in $H_{\varphi}^{1}\left(\Omega, S^{2}\right)$. Then $u$ is smooth $\left(C^{\infty}\right)$ except at a finite number of points in $\Omega$.

A very general partial regularity result asserts that if $u$ is a minimizing harmonic map from $M^{n}$ to $N^{k}$, then the singular set $\Sigma$ of $u$ has Hausdorff dimension $\leq(n-3)$. When $n=3$, the result is more precise: $\Sigma$ consists of a finite number of points.

Shortly afterwards we investigated the shape of $u$ near its singular points and proved

Theorem 3 (H. Brezis, J.-M. Coron and E. Lieb [18]). Assume $u$ is a minimizer of $E$ in $H_{\varphi}^{1}\left(\Omega, S^{2}\right)$ and let $a \in \Omega$ be one of its singular points; then, as $x \rightarrow a$,

$$
u(x) \sim \pm R\left(\frac{x-a}{|x-a|}\right)
$$

for some rotation $R$.

From the simple form of each singularity $a_{i}$ we deduce that

$$
\operatorname{deg}\left(u, a_{i}\right)= \pm 1
$$

By standard degree consideration we have

$$
\operatorname{deg}(\varphi)=\sum_{i} \operatorname{deg}\left(u, a_{i}\right)
$$

and therefore

$$
|\operatorname{deg}(\varphi)| \leq \text { Number of singularities of } u \text {. }
$$

This provides a lower bound for the number of singularities in terms of topological invariants. An interesting open direction is to obtain upper bounds for the number of singularities. The argument of Schoen-Uhlenbeck is too indirect to provide an explicit bound. An interesting estimate of F. Almgren and E. Lieb $\sqrt{2}$ asserts that

$$
\text { Number of singularities of } u \leq C \int_{\partial \Omega}|\nabla \varphi|^{2}
$$

for some universal constant $C$. Again, the argument is so involved that it is hard to keep track of the constant $C$.

Open Problem 1. Evaluate the best constant $C$ in (1.7). Is it $C=1 / 8 \pi$ ?

Note that $1 / 8 \pi$ is a natural candidate in view of the estimate

$$
|\operatorname{deg}(\varphi)| \leq \frac{1}{8 \pi} \int_{\partial \Omega}|\nabla \varphi|^{2} .
$$

A special case of Open Problem 1 is whether the condition $\int_{\partial \Omega}|\nabla \varphi|^{2}<8 \pi$ implies the smoothness of minimizers.

A major difference between the standard Laplace equation (0.1) and the equation of harmonic maps, e.g. (1.2), is that weak solutions of (0.1) are smooth while (1.2) seems to carry little information about the singular set of $u$ (this is true in 3 -d; however, the situation is quite different in 2-d; see Theorem 10 in Section 2). A striking construction of $\mathrm{T}$. Rivière yields: 
Theorem 4 (T. Rivière [51]). Given any smooth $\varphi, \varphi \neq$ Constant, there exists a weak solution $u \in H_{\varphi}^{1}\left(\Omega, S^{2}\right)$ of (1.2) such that

$$
\text { Sing }(u)=\bar{\Omega}
$$

where Sing $(u)$ is the complement of the maximal open set in $\Omega$ on which $u$ is continuous.

In fact, there seems to be a plethora of weakly harmonic maps (see also the construction in Section 1.2) and a natural question is

Open Problem 2. Given any closed set $\Sigma \subset \Omega$, does there exist a weak solution $u \in H_{\varphi}^{1}\left(\Omega, S^{2}\right)$ of $(1.2)$, e.g. for some smooth $\varphi$, such that

$$
\text { Sing }(u)=\Sigma \text { ? }
$$

Let us mention that there is an "intermediate" concept between minimizing harmonic maps and weakly harmonic maps. This is the notion of stationary harmonic maps: they are critical points of the energy $E$ with respect to variations in the domain $\Omega$. Partial regularity results for such maps have been obtained by L. C. Evans [26] and F. Bethuel 4].

1.2. The case where $\operatorname{deg}(\varphi)=0$; minimal connections and relaxed energy. Throughout this section we assume that $\varphi: \partial \Omega=S^{2} \rightarrow S^{2}$ is smooth and that

$$
\operatorname{deg}(\varphi)=0 .
$$

Here, there is no topological obstruction to the smoothness of minimizers, and the initial guess would be that minimizers are smooth: why would they create a singularity at a "high energy cost" when they are not forced by the topology of $\varphi$ ? It turns out that the intuition is wrong! Sometimes configurations with singularities have lower energy than smooth ones (for maps $u \in H_{\varphi}^{1}\left(\Omega, S^{2}\right)$ ), i.e., singularities may save energy! This remarkable phenomenon was first pointed out by R. Hardt and F. H. Lin:

Theorem 5 (R. Hardt and F. H. Lin [35; see also H. Brezis [13). There exist smooth boundary data $\varphi: \partial \Omega \rightarrow S^{2}$ satisfying (1.9) such that any minimizing harmonic map in $H_{\varphi}^{1}\left(\Omega, S^{2}\right)$ must have singularities.

In fact the construction shows that for any $\varepsilon>0$ there exists a smooth $\varphi=\varphi_{\varepsilon}$ : $\partial \Omega \rightarrow S^{2}$ satisfying (1.9) such that

$$
\operatorname{Min}_{u \in H_{\varphi}^{1}\left(\Omega, S^{2}\right)} \int|\nabla u|^{2}<\varepsilon,
$$

while for every $v \in H_{\varphi}^{1}\left(\Omega, S^{2}\right) \cap C^{0}\left(\bar{\Omega}, S^{2}\right)$ we have

$$
\int_{\Omega}|\nabla v|^{2} \geq 16 \pi-\varepsilon
$$

Such an occurrence is sometimes called a gap phenomenon because

$$
\operatorname{Inf}_{u \in H_{\varphi}^{1}\left(\Omega, S^{2}\right)} E(u)<\operatorname{Inf}_{u \in C_{\varphi}^{1}\left(\bar{\Omega}, S^{2}\right)} E(u) .
$$

An intriguing open problem in this kind of situation is

Open Problem 3. Given any smooth $\varphi: \partial \Omega=S^{2} \rightarrow S^{2}$ with $\operatorname{deg}(\varphi)=0$, does there exist a smooth harmonic map satisfying $u=\varphi$ in $\partial \Omega$ ? 
A program to tackle Open Problem 3 was proposed by F. Bethuel, H. Brezis and J.-M. Coron [7]. We have not been able to complete the program, but I feel that there are some tools which are useful in other contexts and I would like to present them here; they involve the notion of relaxed energy and also of minimal connection.

The first observation is that $H_{\varphi}^{1}\left(\Omega, S^{2}\right) \cap C^{0}\left(\bar{\Omega}, S^{2}\right)$ is not dense in $H_{\varphi}^{1}$ for the strong $H^{1}$ topology. This is an immediate consequence of the gap phenomenon (1.10) - (1.11). (A similar observation, without the boundary condition, had been made earlier by R. Schoen and K. Uhlenbeck [56.) There is, however, density for the weak topology :

Lemma 1 (F. Bethuel 4, [5]). Given any $u \in H_{\varphi}^{1}\left(\Omega, S^{2}\right)$ there exists a sequence $v_{n} \in H_{\varphi}^{1}\left(\Omega, S^{2}\right) \cap C^{0}\left(\bar{\Omega}, S^{2}\right)$ such that

$$
v_{n} \rightarrow u \text { weakly in } H^{1} \text {. }
$$

We define the relaxed energy of any $u \in H_{\varphi}^{1}\left(\Omega, S^{2}\right)$ to be

$$
\begin{aligned}
& E_{\mathrm{rel}}(u)= \\
& \quad \operatorname{Inf}\left\{\liminf _{n \rightarrow \infty} \int_{\Omega}\left|\nabla v_{n}\right|^{2} ; v_{n} \in H_{\varphi}^{1}\left(\Omega, S^{2}\right) \cap C^{0}\left(\bar{\Omega}, S^{2}\right) \text { and } v_{n} \rightarrow u \text { weakly } H^{1}\right\} .
\end{aligned}
$$

The concept of relaxed energy plays a very natural role in the Calculus of Variations (see e.g. M. Giaquinta, G. Modica and J. Souček [30]). Clearly

$$
E_{\text {rel }}(u) \geq E(u) \quad \forall u \in H_{\varphi}^{1}\left(\Omega, S^{1}\right) .
$$

It is a pleasant surprise that the relaxed energy $E_{\text {rel }}$ has an explicit form involving the "topological singularities" of $u$. More precisely, given any $u$ in $H_{\varphi}^{1}\left(\Omega, S^{2}\right)$, consider the vector field defined in local coordinates by

$$
D(u)=\left(u \cdot u_{y} \wedge u_{z}, u \cdot u_{z} \wedge u_{x}, u \cdot u_{x} \wedge u_{y}\right)
$$

(this vector field has been introduced in H. Brezis, J.-M. Coron and E. Lieb [18]) and the distribution $T(u)$ defined through its action on $C^{\infty}(\bar{\Omega}, \mathbb{R})$-functions by the formula

$$
<T(u), \zeta>=-\int_{\Omega} D(u) \cdot \nabla \zeta+\int_{\partial \Omega}(\operatorname{Jac} \varphi) \zeta .
$$

Note that the distribution $T(u)$ acts, in fact, on Lip $(\Omega, \mathbb{R})$-functions and also that

$$
D(u) \cdot n=\operatorname{Jac} \varphi \text { on } \partial \Omega \text {. }
$$

Define the nonnegative number

$$
L(u)=\operatorname{Max}_{\substack{\zeta \in \operatorname{Lip}(\Omega, \mathbb{R}) \\\|\nabla \zeta\|_{L} \infty \leq 1}}<T(u), \zeta>
$$

and observe that $L(u)<\infty$ since

$$
\int_{\partial \Omega}(\operatorname{Jac} \varphi)=4 \pi \operatorname{deg}(\varphi)=0 .
$$


Theorem 6 (F. Bethuel, H. Brezis and J.-M. Coron [7]). We have, for every $u \in H_{\varphi}^{1}\left(\Omega, S^{2}\right)$,

$$
E_{r e l}(u)=E(u)+8 \pi L(u) .
$$

The distribution $T(u)$ and the number $L(u)$ have interesting interpretations in terms of "topological singularities".

A straightforward computation (see H. Brezis, J.-M. Coron and E. Lieb [18]) shows that if $u \in C^{2}\left(\bar{\Omega}, S^{2}\right)$, then $T(u)=0$. More generally, if $u \in H_{\varphi}^{1}\left(\Omega, S^{2}\right)$ and $u \in C^{0}\left(\bar{\Omega} \backslash \bigcup_{i-1}^{m}\left\{a_{i}\right\}\right)$, then

$$
T(u)=4 \pi \sum d_{i} \delta_{a_{i}}
$$

where $a_{1}, a_{2}, \ldots, a_{m}$ are $m$ points in $\Omega$, and $d_{i}=\operatorname{deg}\left(u, a_{i}\right)=$ topological degree of $u$ restricted to any small sphere around $a_{i}$. Here, the relation (1.19) is to be interpreted in the sense of distributions, i.e.,

$$
-\int_{\Omega} D(u) \cdot \zeta+\int_{\partial \Omega}(\operatorname{Jac} \varphi) \zeta=4 \pi \sum_{i} d_{i} \zeta\left(a_{i}\right)
$$

for every $\zeta \in \operatorname{Lip}(\Omega, \mathbb{R})$.

The distribution $T(u)$ is a simple but efficient tool which carries information about the location and the topological strength of the singularities. Note that $T$ detects only "topological singularities". For example an "analytic singularity" such as

$$
u(x, y, z)=\left(\cos \frac{1}{r^{\alpha}} \sin \frac{1}{r^{\beta}}, \sin \frac{1}{r^{\alpha}} \sin \frac{1}{r^{\beta}}, \cos \frac{1}{r^{\beta}}\right),
$$

with $r^{2}=x^{2}+y^{2}+z^{2}, 0<\alpha<1 / 2,0<\beta<1 / 2$, goes undetected and $T(u)=0$.

Since $u=\varphi$ on $\partial \Omega$ we have

$$
\sum d_{i}=-\operatorname{deg}(\varphi)=0 .
$$

The points $a_{i}$ with $d_{i}>0$ (resp. $\left.d_{i}<0\right)$ are called positive (resp. negative). We list the positive points with each $a_{i}$ repeated $d_{i}$ times in the list. Write this list as $p_{1}, p_{2}, \ldots, p_{k}$, with each $p_{j}$ one of the positive points. Likewise, list the negative points and write them as $n_{1}, n_{2}, \ldots, n_{k}$. The points of degree zero, i.e., the "nontopological singularities", are omitted from these two lists. Rewriting (1.19) we may now state

Theorem 7. Assume $u \in H_{\varphi}^{1}\left(\Omega, S^{2}\right)$ and $u$ is continuous on $\bar{\Omega}$ except at a finite number of points in $\Omega$. Then

$$
T(u)=4 \pi\left(\sum_{i=1}^{k} \delta_{p_{i}}-\sum_{i=1}^{k} \delta_{n_{i}}\right),
$$

and $L(u)$, defined by (1.17), can be represented as

$$
L(u)=\underset{\sigma}{\operatorname{Min}} \sum_{i=1}^{k}\left|p_{i}-n_{\sigma(i)}\right|
$$

where the minimum in (1.22) is taken over all permutations $\sigma$ of the integers $\{1,2, \ldots, k\}$. 
Formula (1.22) is an immediate consequence of (1.17), (1.21), and the following general

Lemma 2 (H. Brezis, J.-M. Coron and E. Lieb [18]). Assume $X$ is a metric space. Let $p_{1}, p_{2}, \ldots, p_{k}$ and $n_{1}, n_{2}, \ldots, n_{k}$ be $2 k$ points in $X$.

Then

$$
\begin{aligned}
\underset{\zeta}{\operatorname{Max}} & \left\{\sum_{i=1}^{k}\left(\zeta\left(p_{i}\right)-\zeta\left(n_{i}\right)\right) ; \zeta \in \operatorname{Lip}(\Omega, \mathbb{R}) \text { and }|\zeta|_{\text {Lip }} \leq 1\right\} \\
& =\underset{\sigma}{\operatorname{Min}} \sum_{i=1}^{k} d\left(p_{i}, n_{\sigma(i)}\right),
\end{aligned}
$$

where $|\zeta|_{\text {Lip }}=\underset{x \neq y}{\operatorname{Sup}|\zeta(x)-\zeta(y)| / d(x, y) .}$

$L$ is called the length of a minimal connection connecting the positive points to the negative points.

Note that the distribution $T(u)$ and the number $L(u)$ are well-defined, through the formulas (1.15) and (1.17), for every $u \in H_{\varphi}^{1}\left(\Omega, S^{2}\right)$. And then one has the following:

Theorem $7^{\prime}$ (H. Brezis and P. Mironescu). Given any $u \in H_{\varphi}^{1}\left(\Omega, S^{2}\right)$, there exist two infinite sequences $p_{1}, p_{2}, \ldots$ and $n_{1}, n_{2}, \ldots$ in $\Omega$ such that

$$
\sum_{i=1}^{\infty}\left|p_{i}-n_{i}\right|<\infty
$$

and

$$
T(u)=4 \pi \sum_{i=1}^{\infty}\left(\delta_{p_{i}}-\delta_{n_{i}}\right) .
$$

Note that the right hand side in (1.25) is not a measure but a distribution acting on Lip $(\Omega, \mathbb{R})$ through the formula

$$
<\sum_{i}\left(\delta_{p_{i}}-\delta_{n_{i}}\right), \zeta>=\sum_{i}\left(\zeta\left(p_{i}\right)-\zeta\left(n_{i}\right)\right) .
$$

The proof follows closely an argument in J. Bourgain, H. Brezis and P. Mironescu [10] (see Theorem 1). Moreover, $L(u)$ (defined by (1.17)) has an interpretation in terms of a minimal connection:

$$
\begin{aligned}
L(u) & =\operatorname{Max}_{\zeta}\left\{\sum_{i=1}^{\infty}\left(\zeta\left(p_{i}\right)-\zeta\left(n_{i}\right)\right) ;|\zeta|_{\text {Lip }} \leq 1\right\} \\
& =\operatorname{Inf}_{\left(\tilde{p}_{i}\right),\left(\tilde{n}_{i}\right)}\left\{\sum_{i=1}^{\infty}\left|\tilde{p}_{i}-\tilde{n}_{i}\right| ; T(u)=\sum_{i=1}^{\infty}\left(\delta_{\tilde{p}_{i}}-\delta_{\tilde{n}_{i}}\right)\right\} .
\end{aligned}
$$

Useful related results are:

Theorem 8 (F. Bethuel [4], 5], F. Bethuel and X. Zheng 9]). Let $\mathcal{R}$ denote the class of maps in $H_{\varphi}^{1}\left(\Omega, S^{2}\right)$ which are smooth on $\bar{\Omega}$, except at a finite number of points. Then $\mathcal{R}$ is dense in $H_{\varphi}^{1}$ (in the strong $H^{1}$ topology).

Theorem 9 (F. Bethuel [5]). Let $u \in H_{\varphi}^{1}\left(\Omega, S^{2}\right)$. Then $L(u)=0$ if and only if there is a sequence $\left(u_{n}\right)$ in $C_{\varphi}^{1}\left(\bar{\Omega}, S^{2}\right)$ such that $u_{n} \rightarrow u$ in $H^{1}$. 
One may also ask whether a kind of "converse" to Theorem $7^{\prime}$ holds:

Open Problem 4. Given any sequence of points $\left(p_{i}\right),\left(n_{i}\right)$ in $\Omega$ with $\sum_{i=1}^{\infty}\left|p_{i}-n_{i}\right|<\infty$, can one find some $u \in H_{\varphi}^{1}\left(\Omega, S^{2}\right)$ such that

$$
T(u)=4 \pi \sum_{i=1}^{\infty}\left(\delta_{p_{i}}-\delta_{n_{i}}\right) ?
$$

What is the optimal bound for $\int|\nabla u|^{2}$ in terms of $\varphi$ and the length of a minimal connection $L=\operatorname{Max}_{\zeta}\left\{\sum_{i=1}^{\infty}\left(\zeta\left(p_{i}\right)-\zeta\left(n_{i}\right)\right) ;|\zeta|\right.$ Lip $\left.\leq 1\right\}$ ?

The dipole construction introduced in H. Brezis, J.-M. Coron and E. Lieb [18] (see also F. Bethuel [5] and T. Rivière [51]) should be a basic ingredient in the construction of $u$.

We refer to M. Giaquinta, G. Modica and J. Souček [30], R. L. Jerrard and H. M. Soner [1], and G. Alberti, S. Baldo and G. Orlandi [1] for related questions in a more general geometric setting.

We now return to Open Problem 3 . The first observation is that $E_{\text {rel }}$ (defined by (1.13) or by (1.18)) is lower-semicontinuous (l.s.c.) for the weak topology on $H_{\varphi}^{1}$. Therefore,

$$
\operatorname{Min}_{u \in H_{\varphi}^{1}\left(\Omega, S^{2}\right)} E_{\mathrm{rel}}(u) \quad \text { is achieved. }
$$

In fact, for every $t \in[0,1]$ the functional

$$
E_{t}(u)=\int_{\Omega}|\nabla u|^{2}+8 \pi t L(u)
$$

defined on $H_{\varphi}^{1}\left(\Omega, S^{2}\right)$ is l.s.c. for the weak topology on $H_{\varphi}^{1}$, and thus

$$
\operatorname{Min}_{u \in H_{\varphi}^{1}\left(\Omega, S^{2}\right)} E_{t}(u) \quad \text { is achieved. }
$$

Moreover, any critical point of $E_{t}$ on $H_{\varphi}^{1}\left(\Omega, S^{2}\right)$ (and in particular any minimizer) is a weakly harmonic map; i.e., it satisfies (1.2). In particular, this machinery allows one (in general) to construct a family of weakly harmonic maps with a given boundary condition $\varphi$. When $t=0$, the minimizers of $E_{0}=E$ usually have singularities. One may hope that by "turning on" the $L$-term in $E_{t}$, the singularities of opposite signs will attract each other and eventually coalesce as $t \rightarrow 1$. Unfortunately, little is known about the regularity of minimizers for $E_{t}$.

Open Problem 5. Let $u$ be a minimizer for $E_{t}, 0<t<1$, in $H_{\varphi}^{1}\left(\Omega, S^{2}\right)$. Is $u$ smooth in $\bar{\Omega}$ except at a finite number of points in $\Omega$ ? Let $u$ be a minimizer for $E_{1}$. Is $u$ smooth in $\bar{\Omega}$ ? (Try first to show that $L(u)=0$.)

Partial regularity results for $E_{t}$ are discussed in M. Giaquinta, G. Modica and J. Souček [30].

\section{Model Case II: $M=B^{2}$ And $N=S^{2}$}

In this section we take $\Omega=M=B^{2}$, the unit disc in $\mathbb{R}^{2}$, and $N=S^{2}$, the unit sphere in $\mathbb{R}^{3}$. Given a smooth boundary condition $\varphi: \partial \Omega=S^{1} \rightarrow S^{2}$, we 
investigate the minimization problem

$$
\operatorname{Min}_{\substack{u: \Omega \rightarrow S^{2} \\ u=\varphi \text { in } \partial \Omega}} E(u), \text { where } E(u)=\int_{\Omega}|\nabla u|^{2} .
$$

There are two major differences with Model Case I:

1) Here, there is no topological obstruction to regularity. For any smooth Dirichlet data $\varphi$, the class $C_{\varphi}^{\infty}\left(\bar{\Omega}, S^{2}\right)$ is nonempty; the topological condition $\operatorname{deg}(\varphi)=0$ encountered in Section 1 has no counterpart.

2) A remarkable result of F. Hélein asserts that any weak solution $u \in H_{\varphi}^{1}\left(\Omega, S^{2}\right)$ of (1.2) is smooth. This is true in a much more general setting: the target $N$ can be a manifold (of any dimension) provided the domain $\Omega$ is 2-dimensional.

Theorem 10 (F. Hélein [37], 38]). Assume $u \in H_{\varphi}^{1}(\Omega, N)$ is a weakly harmonic map, where $N$ is any Riemannian manifold. Then $u$ is smooth.

Remark 1. The regularity result of F. Hélein is very delicate and sensitive to the special structure of the harmonic map equation (1.2) (or (0.3)). For other systems similar in appearance to (1.2) - with quadratic growth in $\nabla u$ - the same conclusion might fail: weak $H^{1}$ solutions are not smooth; see e.g. S. Hildebrandt [40]. There seems to be some kind of compensation phenomenon, possibly similar to the nullcondition of S. Klainerman; see e.g. S. Klainerman and M. Machedon [44, 45]. Another well-known equation which has the property that weak $H^{1}$ solutions are smooth is the system $\Delta u=u_{x} \wedge u_{y}$ occurring in the study of surfaces of constant mean curvature (see Wente [59], H. Brezis and J.-M. Coron [17]). An interesting direction of research is

Open Problem 6. Let $u \in H^{1}(\Omega, N)$ be a weak solution of

$$
-\Delta u=F(x, u, \nabla u) \text { in } \Omega \subset \mathbb{R}^{2} .
$$

For which nonlinearities $F$ can one conclude that $u$ is smooth?

Here, one of the intriguing directions of research is the existence of multiple solutions for (1.2). Such a question was originally raised by M. Giaquinta and S. Hildebrandt [29]. Clearly,

$$
\operatorname{Min}_{u \in H_{\varphi}^{1}\left(\Omega, S^{2}\right)} \int|\nabla u|^{2} \quad \text { is achieved, }
$$

and the range $u(\Omega)$ of a minimizer lies in the "small" spherical cap on $S^{2}$ enclosed by the curve $\varphi(\partial \Omega)$. M. Giaquinta and S. Hildebrandt asked whether there is more than one solution to (1.2). The answer is indeed positive.

Theorem 11 (H. Brezis and J.-M. Coron [16], J. Jost [42]). Given any $\varphi \not \equiv$ constant, problem (1.2) admits at least two distinct solutions.

The technique we used in H. Brezis and J.-M. Coron 16 illustrates (in a rather elementary setting) the need to work with homotopy classes within the framework of Sobolev spaces (more about this in Section 4). The heart of the matter is

Lemma 3. The space $H_{\varphi}^{1}\left(\Omega, S^{2}\right)$ admits infinitely many connected components. 
In order to construct these components we use a "relative" degree. Fix a "reference" map $u_{0} \in H_{\varphi}^{1}\left(\Omega, S^{2}\right)$, for example a minimizer for (2.1). Given any $u \in H_{\varphi}^{1}\left(\Omega, S^{2}\right)$ set

$$
v=\left(\begin{array}{c}
u \\
u_{0}
\end{array}\right): S^{2} \rightarrow S^{2}
$$

here we identify one copy of $\Omega$ with the upper hemisphere $S_{+}^{2}$ and another copy of $\Omega$ with lower hemisphere $S_{-}^{2}$. The two maps $u$ and $u_{0}$ glue well since they agree on the hemisphere and thus $v \in H^{1}\left(S^{2}, S^{2}\right)$. The key point is

Lemma 4. Maps in $H^{1}\left(S^{2}, S^{2}\right)$ have a well-defined degree.

Lemma 4 is far from obvious since maps in $H^{1}\left(S^{2}, S^{2}\right)$ need not be continuous and one cannot rely on standard degree. One way of defining degree is via the classical Kronecker formula

$$
\operatorname{deg}(w)=\frac{1}{4 \pi} \int_{S^{2}} \operatorname{Jac}(w)
$$

for any $w \in C^{1}\left(S^{2}, S^{2}\right)$. If $w \in H^{1}\left(S^{2}, S^{2}\right)$, the right hand side in (2.2) makes sense (since $\operatorname{Jac}(w)$ is the determinant of a $2 \times 2$ matrix with $L^{2}$ entries). An additional density argument due to R. Schoen and K. Uhlenbeck [56] allows one to conclude that the r.h.s. in (2.2) belongs to $\mathbb{Z}$ for every $w \in H^{1}\left(S^{2}, S^{2}\right)$ (see Section 4 ).

One may then decompose $H_{\varphi}^{1}\left(\Omega, S^{2}\right)$ into disjoint classes

$$
H_{\varphi}^{1}\left(\Omega, S^{2}\right)=\bigcup_{k \in \mathbb{Z}} \mathcal{E}_{k}
$$

where

$$
\mathcal{E}_{k}=\left\{u \in H_{\varphi}^{1}\left(\Omega, S^{2}\right) ; \operatorname{deg}\left(\begin{array}{c}
u \\
u_{0}
\end{array}\right)=k\right\} .
$$

It is tempting to minimize the energy in each class $\mathcal{E}_{k}$. Here we encounter a new difficulty which is common in "critical" problems involving "bubbling of spheres" (as in J. Sacks and K. Uhlenbeck [54]): "lack of compactness", etc.; namely, $\operatorname{Inf}_{\mathcal{E}_{k}} E$ need not be achieved, the reason being that a minimizing sequence con$\mathcal{E}_{k}$ verges only weakly in $H^{1}$ but the class $\mathcal{E}_{k}$ defined via $(2.2)$ is not closed under weak convergence. To overcome this difficulty we use a method similar to the one introduced by Th. Aubin [3] for the Yamabe problem. This device works (assuming $\varphi \not \equiv$ Constant) and yields the existence of a minimizer for $E$ in one of the classes $\mathcal{E}_{+1}$ or $\mathcal{E}_{-1}$. The existence of more solutions for the equation (1.2) is not known even when $\varphi$ has a simple explicit form. Consider the Dirichlet data

$$
\varphi(x, y)=\left(R x, R y, \sqrt{1-R^{2}}\right) \text { for }(x, y) \in \partial \Omega
$$

with $0<R<1$. In this case one can write down explicitly two solutions:

$$
\underline{u}(x, y)=\frac{2 \lambda}{\lambda^{2}+r^{2}}(x, y, \lambda)+(0,0,-1)
$$

and

$$
\bar{u}(x, y)=\frac{2 \mu}{\mu^{2}+r^{2}}(x, y,-\mu)+(0,0,1)
$$

with $(x, y) \in \Omega, r^{2}=x^{2}+y^{2}, \lambda=\frac{1}{R}+\sqrt{\frac{1}{R^{2}}-1}$ and $\mu=\frac{1}{R}-\sqrt{\frac{1}{R^{2}}-1}$. 
It is not difficult to see that $\underline{u}$ is the absolute minimizer of $E$ in $H_{\varphi}^{1}\left(\Omega, S^{2}\right)$. Using $\underline{u}$ as the reference map $u_{0}$, we see that $\bar{u} \in \mathcal{E}_{-1}$ and that $\bar{u}$ is the minimizer in the class $\mathcal{E}_{-1}$. In addition, one can show that besides these two, no other minimizer exists in any of the classes $\mathcal{E}_{k}$.

Open Problem 7. Are there other solutions of (1.2) besides $\underline{u}$ and $\bar{u}$ ?

Either way, the answer to Open Problem 7 would be illuminating. A negative answer (only 2 solutions) might possibly shed some light on the important questions of whether solutions of some nonlinear systems inherit the symmetry of the data. A positive answer (more than 2 solutions) might involve the development of new techniques for finding (nonminimizing) critical points in variational problems with lack of compactness.

\section{Model Case III: $M=B^{2}\left(\right.$ or $\left.B^{3}\right)$ And $N=S^{1}$}

In this section we first take $\Omega=M=B^{2}$ and later $\Omega=M=B^{3} . N$ will always be $S^{1}$, the unit circle in $\mathbb{R}^{2}$.

3.1 $M=\Omega=B^{2}$ and $N=S^{1}$. Given a smooth boundary condition $\varphi: \partial \Omega=$ $S^{1} \rightarrow S^{1}$, we investigate the minimization problem

$$
\operatorname{Min}_{\substack{u: \Omega \rightarrow S^{1} \\ u=\varphi \text { on } \partial \Omega}} E(u), \text { where } E(u)=\int_{\Omega}|\nabla u|^{2} .
$$

A new feature occurs, which was not present in Model Cases I and II.

a) If $\operatorname{deg}(\varphi)=0$, there is no topological obstruction: $C_{\varphi}^{\infty}\left(\bar{\Omega}, S^{1}\right)$ is not empty. In this case we will see (in Theorem 12) that problem (3.1) is quite easy.

b) However, if $\operatorname{deg}(\varphi) \neq 0$, there is a topological obstruction to regularity since $C_{\varphi}^{0}\left(\bar{\Omega}, S^{1}\right)$ is empty. In some sense, the situation is "worse" than in Model Case I! Recall that, there, if $\operatorname{deg}(\varphi) \neq 0, C_{\varphi}^{0}\left(\bar{\Omega}, S^{2}\right)$ was empty, but at least the class $H_{\varphi}^{1}\left(\Omega, S^{2}\right)$ was not empty. Here, if $\operatorname{deg}(\varphi) \neq 0$, both $C_{\varphi}^{0}\left(\bar{\Omega}, S^{1}\right)$ and the natural energy class $H_{\varphi}^{1}\left(\Omega, S^{1}\right)$ are empty (see Lemma 5). Thus problem (3.1) makes no sense.

In fact, for many years, this case was considered "off-limits"; to quote R. Hamilton 32]: "If there is a topological obstruction to extending [the boundary condition] $h$, then $\mathcal{M}_{h}(X, Y)$ is empty and nothing more can be said." At the beginning of the 90's the "taboo" was broken. The incentive came from problems in physics, specifically in the theory of superfluids and superconductors. Their mathematical modeling resembles (3.1), and the "solutions" in the physics literature involve point vortices (in $2-d$ ) or line vortices (in $3-d$ ). The new idea is to tackle the "impossible" problem (3.1) via a Ginzburg - Landau mechanism; namely, the constraint $u: \Omega \rightarrow S^{1}$ (which is at the heart of the topological obstruction) is relaxed and replaced by a Ginzburg - Landau "penalty". More precisely, one considers all functions $u: \Omega \rightarrow \mathbb{R}^{2}$, but the standard energy $\int_{\Omega}|\nabla u|^{2}$ is replaced by the Ginzburg -

\section{Landau energy}

$$
E_{\varepsilon}(u)=\frac{1}{2} \int_{\Omega}|\nabla u|^{2}+\frac{1}{4 \varepsilon^{2}} \int_{\Omega}\left(|u|^{2}-1\right)^{2}
$$


with a small parameter $\varepsilon>0$. The strategy is to study the approximate problem

$$
\operatorname{Min}_{u \in H_{\varphi}^{1}\left(\Omega, \mathbb{R}^{2}\right)} E_{\varepsilon}(u)
$$

and to analyze the limiting behavior of minimizers $u_{\varepsilon}$ as $\varepsilon \rightarrow 0$. This program was initiated in 1992 by F. Bethuel, H. Brezis and F. Hélein [ 8 ] and was followed by many subsequent developments.

Here are some precise statements.

Lemma 5. Let $\varphi: \partial \Omega=S^{1} \rightarrow S^{1}$ be a smooth map. Then

$$
H_{\phi}^{1}\left(\Omega, S^{1}\right) \neq \emptyset \Longleftrightarrow \operatorname{deg}(\varphi)=0
$$

The proof of Lemma 5 involves the $H^{1 / 2}$-degree theory for maps of $S^{1}$ into itself (see H. Brezis and L. Nirenberg [21]); it stresses once more the need to study Homotopy theory in the framework of Sobolev spaces (see Section 4).

When $\operatorname{deg}(\varphi)=0$, we have a complete and simple answer to problem (3.1).

Theorem 12. Given any smooth map $\varphi: \partial \Omega=S^{1} \rightarrow S^{1}$ with $\operatorname{deg}(\varphi)=0$, there exists a unique minimizer $u$ of problem (3.1) - in fact a unique solution $u \in H_{\varphi}^{1}\left(\Omega, S^{1}\right)$ of the corresponding PDE (1.2). More precisely, $u=e^{i \psi}$ where $\psi$ is the solution of

$$
\begin{aligned}
\Delta \psi & =0 & & \text { in } \Omega \\
\psi & =\psi_{0} & & \text { on } \partial \Omega
\end{aligned}
$$

and $\psi_{0}$ is a smooth lifting of $\varphi$, i.e., $e^{i \psi_{0}}=\varphi$ on $\partial \Omega$.

The proof of Theorem 12 relies heavily on the theory of lifting for Sobolev maps into $S^{1}$ initiated by F. Bethuel and X. Zheng [9] and completed by J. Bourgain, H. Brezis and P. Mironescu 10].

When $\operatorname{deg}(\varphi) \neq 0$ the situation is much more complicated. Here is a typical result:

Theorem 13 (F. Bethuel, H. Brezis and F. Hélein [8]). For a subsequence $\varepsilon_{n} \rightarrow$ $0, u_{\varepsilon_{n}} \rightarrow u_{*}$ pointwise on $\Omega$, except at a finite number of distinct points $a_{1}, a_{2}, \ldots, a_{d}$ in $\Omega$, where $d=|\operatorname{deg}(\varphi)|$. Moreover $u_{*}$ is smooth on $\bar{\Omega}$, except at the points $\left(a_{i}\right),\left|u_{*}\right|=1$ on $\Omega$, and $u_{*}$ satisfies the equation of harmonic maps (1.2) on $\Omega \backslash \bigcup_{i=1}^{d}\left\{a_{i}\right\}$. Moreover, if $\operatorname{deg}(\varphi)>0$, then, near each $a_{i}$,

$$
u_{*}(x) \sim R \frac{\left(x-a_{i}\right)}{\left|x-a_{i}\right|} \text { as } x \rightarrow a_{i}
$$

for some rotation $R$.

The limit $u_{*}$ can be viewed as a "minimizing harmonic map" in some generalized sense even though its energy $\int_{\Omega}\left|\nabla u_{*}\right|^{2}$ is infinite. In fact $\int_{\Omega}\left|\nabla u_{\varepsilon}\right|^{2} \sim 2 \pi d \log (1 / \varepsilon)$ as $\varepsilon \rightarrow 0$. Infinite energies do not seem to bother physicists: one can "renormalize" the energy and keep only a finite quantity which plays an important role in locating the position of the singularities. We refer to the book of F. Bethuel, H. Brezis and F. Hélein 8] for a detailed discussion. We also point out that formula (3.3) implies

$$
\operatorname{deg}\left(u_{*}, a_{i}\right)=+1 \quad \forall i
$$

if $\operatorname{deg}(\varphi)>0$ (otherwise $\operatorname{deg}\left(u_{*}, a_{i}\right)=-1, \forall i$ ). Property (3.4) is consistent with the physical observation that all vortices have the same orientation. This is in contrast 
to the situation described in Section 1, where singularities of opposite orientation do coexist. In view of (3.4) the count of the number of singularities is very easy.

Another important feature is that the limit $u_{*}$ seems to be a kind of "canonical" solution for the "impossible" problem (3.1). It is in some sense "intrinsic" and does not depend on the Ginzburg-Landau approximation. There are several ways of relaxing the topological obstruction by introducing an $\varepsilon$-approximation: for example by drilling holes of size $\varepsilon$ in $\Omega$, or by considering the energy $\int_{\Omega}|\nabla u|^{2-\varepsilon}$. They all yield the same limit $u_{*}$ (see F. Bethuel, H. Brezis and F. Hélein [8], and R. Hardt and F. H. Lin [36]).

3.2 $M=\Omega=B^{3}$ and $N=S^{1}$. Here one investigates problem (3.1) with a boundary condition $\varphi: \partial \Omega=S^{2} \rightarrow S^{1}$ having singularities. An important example is the case where $\varphi$ is smooth on $\partial \Omega$ except at a finite number of singularities $a_{i}$ on $\partial \Omega$, and that, near each $a_{i}$,

$$
\varphi(z) \sim\left(\frac{\left(z-a_{i}\right)}{\left|z-a_{i}\right|}\right)^{d_{i}}, \quad z \in \partial \Omega
$$

modulo rotation. For simplicity one assumes that $\partial \Omega$ is flat near each $a_{i}$, so that the right hand side in (3.5) belongs to $S^{1}$. Clearly

$$
\sum d_{i}=0
$$

and hence, one can list the points $\left(a_{i}\right)$ as $\left(p_{i}\right),\left(n_{i}\right)$ (see Section 1). Here again one can prove that the natural energy class $H_{\varphi}^{1}\left(\Omega, S^{1}\right)$ is empty (this is already true locally, near a singularity; once more the proof uses the $H^{1 / 2}$-degree theory for maps of $S^{1}$ into itself). So that problem (3.1) is impossible, and it is approached via the Ginzburg-Landau mechanism. The main result is the following:

Theorem 14 (T. Rivière 52, F. H. Lin and T. Rivière 47]). For a subsequence $\varepsilon_{n} \rightarrow 0, u_{\varepsilon_{n}} \rightarrow u_{*}$ pointwise on $\Omega$, except along a minimal connection $\Sigma$ connecting in $\Omega$ the points $p_{i}$ to the points $n_{i}$. Moreover $u_{*}$ is smooth in $\bar{\Omega} \backslash \Sigma$ and $u_{*}$ satisfies the equation of harmonic maps (1.2) in $\Omega \backslash \Sigma$.

J. Bourgain, H. Brezis and P. Mironescu [12] have investigated the case of a general boundary condition $\varphi \in H^{1 / 2}\left(\partial \Omega, S^{1}\right)$.

An interesting

Open Direction 8. Investigate other situations (for general manifolds $M$ and $N$ ) where the boundary condition $\varphi: \partial M \rightarrow N$ generates an obstruction and where the natural energy space $H_{\varphi}^{1}(M, N)$ is empty.

\section{Homotopy theory in the Framework of Sobolev Spaces}

Let $M$ and $N$ be two compact Riemannian manifolds. One of the central questions in Topology is the study of homotopy classes of $C^{0}(M, N)$, i.e., the connected (or equivalently path-connected) components of the metric space $C^{0}(M, N)$. In other words, homotopy classes are the equivalence classes corresponding to the equivalence relation: $f \sim g$ if there exists a path $h(t) \in C^{0}\left([0,1] ; C^{0}(M, N)\right)$ connecting $f$ to $g$, i.e., $h(0)=f$ and $h(1)=g$. For example, when $M=N=S^{n}$, it is well-known that $f \sim g$ if and only if $\operatorname{deg}(f)=\operatorname{deg}(g)$. 
In this section, I would like to present some new results concerning the study of homotopy classes when the space $C^{0}(M, N)$ is replaced by the Sobolev space $W^{1, p}(M, N)$.

Throughout this section $M$ and $N$ are smooth, connected, compact, oriented, Riemannian manifolds. We will always assume that $\partial N=\phi$, but $M$ may or may not have a boundary; in particular the case where $M$ is a domain in $\mathbb{R}^{n}$ is of interest. The reader is encouraged to keep in mind elementary manifolds, such as spheres, balls and their products; the results are already of interest for such a simple situation.

Let us start with a simple observation about the scale of spaces $C^{k}(M, N)$ equipped with the metric

$$
d(f, g)=\sum_{|\alpha| \leq k} \sup _{x \in M} d\left(D^{\alpha} f(x), D^{\alpha} g(x)\right) .
$$

In principle, for each $k$, one may introduce a new equivalence relation:

$f \sim g$ in $C^{k} \Longleftrightarrow f, g$ belong to the same path-connected component of $C^{k}$;

i.e., there exists a homotopy $h(t) \in C\left([0,1] ; C^{k}(M, N)\right)$ such that $h(0)=f$ and $h(1)=g$.

In fact, such a notion has no interest because of the "standard"

Lemma 6. Let $f, g \in C^{k}(M, N)$; then $f \sim g$ in $C^{k}$ if and only if $f \sim g$ in $C^{0}$.

The proof consists of smoothing the given homotopy $h \in C\left([0,1] ; C^{0}(M, N)\right)$. For this purpose we may assume that $N \subset \mathbb{R}^{K}$ is an isometric embedding. Then $\rho_{\varepsilon} \star h$ does not take its values into $N$, but $\rho_{\varepsilon} \star h$ is uniformly close to $h$ as $\varepsilon \rightarrow 0$ (because $h$ is continuous). And then one may project $\rho_{\varepsilon} \star h$ back onto $N$ for $\varepsilon$ small.

As a consequence of Lemma 6 we see that the components of $C^{k}(M, N)$ shrink as $k$ increases, but they "do not change their shape". By contrast, we will see that the situation is totally different in the scale of Sobolev spaces $W^{1, p}$.

Let $1 \leq p<\infty$ be a real number. Let $M$ and $N$ be as above and $N \subset \mathbb{R}^{K}$. The spaces $W^{1, p}(M, \mathbb{R})$ and $W^{1, p}\left(M, \mathbb{R}^{K}\right)$ are defined as usual and equipped with the standard norm $\|f\|_{W^{1, p}}$. Set

$$
W^{1, p}(M, N)=\left\{f \in W^{1, p}\left(M, \mathbb{R}^{K}\right) ; f(x) \in N \text { a.e. }\right\},
$$

equipped with the distance

$$
d(f, g)=\|f-g\|_{W^{1, p}\left(M, \mathbb{R}^{K}\right)} .
$$

As a metric space, $W^{1, p}(M, N)$ admits connected components and also pathconnected components. In fact, they coincide because of the following result which is implicit in the work of F. B. Hang and F. H. Lin 33.

Proposition 1. Given $f \in W^{1, p}(M, N)$, there exists $\varepsilon>0$ (depending on $f$ ) such that

$$
d(g, f)<\varepsilon \Longrightarrow g \sim f \text { in } W^{1, p}(M, N) .
$$

We say that $g \sim f$ in $W^{1, p}$ if there is a path $h(t) \in C\left([0,1] ; W^{1, p}(M, N)\right)$ such that $h(0)=f$ and $h(1)=g$.

A simple but useful example is the case $M=N=S^{2}$ which was already mentioned in Section 2. More generally, let us examine the space $W^{1, p}\left(S^{2}, S^{2}\right)$, $1 \leq p<\infty$, from the point of view of its components. One has to consider 3 different cases: 
a) Case $p>2$. It is not difficult to prove, following the same idea as in Lemma 6 and using the Sobolev embedding, $W^{1, p}\left(S^{2}, S^{2}\right) \subset C^{0}\left(S^{2}, S^{2}\right)$,

Lemma 7. Let $f, g \in W^{1, p}\left(S^{2}, S^{2}\right)$; then $f \sim g$ in $W^{1, p}\left(S^{2}, S^{2}\right)$ if and only if $f \sim g$ in $C^{0}$. In particular, the homotopy classes of $W^{1, p}\left(S^{2}, S^{2}\right)$ can be classified using their standard degree.

b) Case $p=2$. This is a very interesting case because it is a limiting case for the Sobolev embedding. $W^{1,2}$ is not contained in $C^{0}$, so that the standard notion of degree is not well-defined. One may nevertheless still define a degree, as we already mentioned in Section 2, using the following strategy:

Step 1. If $f \in C^{1}\left(S^{2}, S^{2}\right)$, Kronecker's formula asserts that

$$
\operatorname{deg}(f)=\frac{1}{4 \pi} \int_{S^{2}} \operatorname{det}(\nabla f) .
$$

When $f \in W^{1,2}\left(S^{2}, S^{2}\right)$ the integral in (4.1) still makes sense. In order to prove that the right-hand side in (4.1) is an integer, one relies on

Step 2. This is an important observation due to Schoen-Uhlenbeck [56]. If $f \in W^{1,2}\left(S^{2}, S^{2}\right)$, then $\rho_{\varepsilon} \star f$ does not take its values into $S^{2}$, and $\rho_{\varepsilon} \star f$ does not converge uniformly to $f$ as $\varepsilon \rightarrow 0$ (otherwise $f$ would be continuous). However, one can prove that $\left|\left(\rho_{\varepsilon} \star f\right)(x)\right| \rightarrow 1$ uniformly in $x$. This is a consequence of the fact that $f \in V M O$ (see H. Brezis and L. Nirenberg 21]) which, in turn, follows from Poincaré's inequality

$$
\int_{B}\left|f-f_{B} f\right| \leq|B|^{1 / 2} \int_{B}|\nabla f|
$$

As a result one may consider

$$
f_{\varepsilon}(x)=P\left(\left(\rho_{\varepsilon} \star f\right)(x)\right)
$$

where $P$ is the projection on $S^{2}$, which is well-defined near $S^{2}$. Then $f_{\varepsilon} \in$ $C^{\infty}\left(S^{2}, S^{2}\right)$ and $f_{\varepsilon} \rightarrow f$ in $W^{1,2}$.

Since $\operatorname{deg}\left(f_{\varepsilon}\right) \in \mathbb{Z}$ and

$$
\operatorname{deg}\left(f_{\varepsilon}\right) \rightarrow \frac{1}{4 \pi} \int_{S^{2}} \operatorname{det}(\nabla f) \text { as } \varepsilon \rightarrow 0
$$

we conclude that

$$
\frac{1}{4 \pi} \int_{S^{2}} \operatorname{det}(\nabla f) \in \mathbb{Z}
$$

We now set, for every $f \in W^{1,2}$

$$
\operatorname{deg}(f)=\frac{1}{4 \pi} \int_{S^{2}} \operatorname{det}(\nabla f) .
$$

It was subsequently observed in H. Brezis and L. Nirenberg [21] that $\operatorname{deg}(f)$ can still be defined for every $f \in W^{1,2}$ without using formula (4.1), only Step 2. Indeed, if $f \in W^{1,2}\left(S^{2}, S^{2}\right)$, consider $f_{\varepsilon}$ as above (for $\varepsilon<\varepsilon_{0}$ ). Then $\operatorname{deg}\left(f_{\varepsilon}\right)$ is independent of $\varepsilon$, for $\varepsilon \in\left(0, \varepsilon_{0}\right)$, because one may use $\varepsilon$ itself as the homotopy parameter, i.e., connect $f_{\varepsilon_{1}}$ and $f_{\varepsilon_{2}}$ via the homotopy $h(t)=f_{t \varepsilon_{1}+(1-t) \varepsilon_{2}}$. We take as the definition of $\operatorname{deg}(f)$ the integer $\operatorname{deg}\left(f_{\varepsilon}\right)$ (for $\left.0<\varepsilon<\varepsilon_{0}\right)$.

Proposition 2. Assume $f, g \in W^{1,2}\left(S^{2}, S^{2}\right)$. Then $f \sim g$ in $W^{1,2}$ if and only if $\operatorname{deg}(f)=\operatorname{deg}(g)$. Consequently, $W^{1,2}\left(S^{2}, S^{2}\right)$ still admits infinitely many homotopy classes and they are classified using degree. 
c) Case $p<2$. This case was not considered in H. Brezis and J.-M. Coron [16]. But in H. Brezis and L. Nirenberg [21] we observed that no degree theory (in any reasonable sense) can be defined. Indeed, the identity map can be homotopied to a constant map! This is done as follows. Fix any point $a \in \mathbb{R}^{3}$ with $|a|=2$. Consider the path

$$
h(t)(x)=\frac{x-t a}{|x-t a|}, \quad t \in[0,1], x \in S^{2} .
$$

Clearly $h$ is smooth for $t \in[0,1 / 2) \cup[1 / 2,1]$ and $x \in S^{2}$. The only difficulty occurs at $t=1 / 2$ because $a / 2 \in S^{2}$. However, it is not difficult to check that $h(1 / 2) \in W^{1, p}\left(S^{2}, S^{2}\right)$ for every $p<2$ and moreover

$$
h(t) \in C\left([0,1] ; W^{1, p}\left(S^{2}, S^{2}\right)\right)
$$

for every $p<2$. Finally it is clear that $h(1)$ has degree zero, and thus it can be homotopied to a constant in the $C^{0}$ sense (and thus in the $C^{1}$ sense by Lemma 6 , and hence in the $W^{1, p}$ sense). Putting all this together we see that $h(0)=I d$ is homotopic to a constant in every $W^{1, p}, p<2$.

We went one step further in H. Brezis and Y. Li [19] and proved

Theorem 15. The space $W^{1, p}\left(S^{2}, S^{2}\right)$ is path-connected for every $p<2$.

We now have a complete picture for $W^{1, p}\left(S^{2}, S^{2}\right)$. When $p<2, W^{1, p}\left(S^{2}, S^{2}\right)$ consists of one piece. At $p=2, W^{1,2}\left(S^{2}, S^{2}\right)$ splits into infinitely many pieces. As $p$ increases from 2 to $\infty$, these pieces "shrink" but "do not change their shape".

Exactly the same type of conclusion holds for $W^{1, p}\left(S^{n}, S^{n}\right)$. When $p<n$, $W^{1, p}\left(S^{n}, S^{n}\right)$ is path-connected. At $p=n$, a degree theory is well-defined, and thus $W^{1, n}\left(S^{n}, S^{n}\right)$ splits into infinitely many pieces. As $p$ increases from $n$ to $\infty$, these pieces shrink without changing their shape.

At this stage, one would be inclined to believe that this phenomenon is typical. When $p<\operatorname{dim} M, W^{1, p}(M, N)$ is path-connected. As $p$ increases from $\operatorname{dim} M$ to $\infty$, $W^{1, p}(M, N)$ admits path-connected components similar to the ones of $C^{0}(M, N)$, and they shrink without changing their shape. The second assertion (for $p \geq$ $\operatorname{dim} M$ ) is indeed true. However, the first assertion (for $p<\operatorname{dim} M$ ) is totally wrong. This was first pointed out in an important paper of B. White 61 and rediscovered a few years later by J. Rubinstein and P. Sternberg 53]: namely, "some topology" still survives for $W^{1, p}(M, N)$, even when $p<\operatorname{dim} M$. In fact $W^{1, p}(M, N)$ may have a very rich structure from the point of view of homotopy classes when $p<\operatorname{dim} M$. I will present later some striking examples.

Theorem 16 (B. White [61], J. Rubinstein and P. Sternberg 53]). Let $M=$ $\Omega=S^{1} \times D$ where $D$ is the unit disc in $\mathbb{R}^{2}$; i.e., $\Omega$ is a solid torus in $\mathbb{R}^{3}$. Let $N=S^{1}$. Then any $f \in W^{1,2}(M, N)$ admits a well-defined degree (stable under $W^{1,2}$ convergence).

More precisely, write

$$
f(x, \lambda): S^{1} \times D \rightarrow S^{1}
$$

then

$$
\varphi(\lambda)=\operatorname{deg} f(\cdot, \lambda),
$$

which is well-defined for a.e. $\lambda \in D$ (since $f(\cdot, \lambda) \in W^{1,2}\left(S^{1}, S^{2}\right)$ for a.e. $\left.\lambda \in D\right)$, is in fact a constant (a.e.). 
Remark 2. It is quite surprising that a degree may still be defined even though $p=2<3=\operatorname{dim} M$. I should point out, however, that the same conclusion fails for $p \in[1,2)$. Note that when $p \in[1,2), \varphi(\lambda)$ is still well-defined for a.e. $\lambda \in D$. However, $\varphi$ is not constant in general.

Remark 3. The conclusion that $\varphi$ is a constant (a.e.) may be related to a variety of results which have emerged in recent years in works of J. Bourgain, H. Brezis and P. Mironescu [10] 11] (see also H. Brezis [14]) about conditions implying that a given measurable function is constant. Here is such a typical result.

Theorem 17. Let $G \subset \mathbb{R}^{N}$ be a connected open set and let $\varphi: G \rightarrow \mathbb{Z}$ be a measurable function. Assume that

$$
\int_{G} \int_{G} \frac{|\varphi(\lambda)-\varphi(\mu)|^{p}}{|\lambda-\mu|^{N+1}} d \lambda d \mu<\infty
$$

(any $p \geq 1$ ). Then $\varphi$ is a constant (a.e.).

An extension of Theorem 16 to higher dimensions is given by

Theorem 18 (H. Brezis, Y. Li, P. Mironescu and L. Nirenberg [20]). Let $M=$ $\Omega=S^{n} \times \Lambda$, where $n \geq 1$, and $\Lambda \subset \mathbb{R}^{k}$ is any open connected set with $k \geq 1$. If $p \geq n+1$, any $f \in W^{1, p}(M, N)$ has a well-defined degree. More precisely, write

$$
f(x, \lambda): S^{n} \times \Lambda \rightarrow S^{n} ;
$$

then

$$
\varphi(\lambda)=\operatorname{deg} f(\cdot, \lambda)
$$

which is well-defined for a.e. $\lambda \in \Lambda$ (since $p>n$ ), is in fact a constant.

Remark 4. Theorem 18 shows again that some topology still "persists" much below the critical Sobolev exponent $p=\operatorname{dim} M$. The condition $p \geq n+1$ is usually much weaker than the condition $p \geq \operatorname{dim} M=n+k$, especially when $k$ is large.

Going back to the study of the path-connected components for $W^{1, p}(M, N)$, here is a complement to Theorem 18, which gives a complete classification of the homotopy classes of $W^{1, p}$ when the parameter space $\Lambda$ is a ball.

Theorem 19 (H. Brezis and Y. Li [19]). Let $M=\Omega=S^{n} \times \Lambda$ where $n \geq 1$ and $\Lambda$ is the unit ball in $\mathbb{R}^{k}$ (any $k \geq 1$ ). Let $f, g \in W^{1, p}(M, N)$. If $p \geq n+1$, then $f \sim g$ in $W^{1, p}$ if and only if $\operatorname{deg}(f)=\operatorname{deg}(g)$ (where deg is meant in the sense of Theorem 18). If $p<n+1$, then, always, $f \sim g$ in $W^{1, p}$; i.e., $W^{1, p}$ is path-connected.

At this stage we decided with Yanyan Li to initiate a general investigation of the homotopy classes of $W^{1, p}(M, N)$ for a general pair of manifolds $M$ and $N$ and for a general $p \geq 1$.

Here is a first (somewhat surprising) result.

Theorem 20 (H. Brezis and Y. Li [19]). For any pair $M$ and $N$ with $\operatorname{dim} M \geq 2$ and for any $p \in[1,2), W^{1, p}(M, N)$ is path-connected.

We also introduced in H. Brezis and Y. Li [19] a concept which plays a very important role in identifying the possible "jumps" in homotopy type for $W^{1, p}$ as $p$ varies. Here it is. 
Let $p>1$. Let $0<\varepsilon<p-1$. Clearly

$$
W^{1, p+\varepsilon}(M, N) \subset W^{1, p-\varepsilon}(M, N) .
$$

Given $f \in W^{1, p}(M, N)$, we denote by $[f]_{p}$ its homotopy class in $W^{1, p}$. We denote by $W^{1, p} / \widetilde{p}$ the quotient of $W^{1, p}$ by the equivalence relation $f_{\widetilde{p}} g$ (meaning $f \sim g$ in $\left.W^{1, p}\right)$.

Obviously, if $f, g \in W^{1, p+\varepsilon}$ and $f \sim g$ in $W^{1, p+\varepsilon}$, then $f \sim g$ in $W^{1, p-\varepsilon}$. As a result we have a canonical map

$$
i_{p+\varepsilon, p-\varepsilon}: W^{1, p+\varepsilon} / \widetilde{p+\varepsilon} \rightarrow W^{1, p-\varepsilon} / \widetilde{p-\varepsilon} .
$$

Definition. We say that a change of topology occurs at $p$ if for every $\varepsilon \in$ $(0, p-1)$, the map $i_{p+\varepsilon, p-\varepsilon}$ is not bijective. We denote by $C T(M, N)$ the set of $p$ 's for which a change of topology occurs at $p$.

If a change of topology occurs at $p$, two things may happen: either $i_{p+\varepsilon, p-\varepsilon}$ is not injective or $i_{p+\varepsilon, p-\varepsilon}$ is not surjective (or both!). Roughly speaking, not injective means that there are 2 maps $f, g \in W^{1, p+\varepsilon}$ such that $f$ and $g$ are not homotopic in $W^{1, p+\varepsilon}$ while $f \sim g$ in $W^{1, p-\varepsilon}$. In other words, one component (at least) of $W^{1, q}(M, N)$ splits into two (or more) distinct components as $q$ increases from $p-\varepsilon$ to $p+\varepsilon$. Another viewpoint is to say that two distinct components of $W^{1, q}$ have coalesced as $q$ decreases from $p+\varepsilon$ to $p-\varepsilon$. This is a common situation, which we have already encountered above. For example, a change of topology for $W^{1, p}\left(S^{2}, S^{2}\right)$ occurs at $p=2$, because $i_{2+\varepsilon, 2-\varepsilon}$ is not injective: any two maps $f, g \in W^{1,2+\varepsilon}$ are homotopic in $W^{1,2-\varepsilon}$, while they need not be in $W^{1,2+\varepsilon}$ (unless their degree is the same).

On the other hand, the fact that $i_{p+\varepsilon, p-\varepsilon}$ is not surjective means that a new component of $W^{1, q}$ appears (out of nowhere!) as $q$ decreases from $p+\varepsilon$ to $p-\varepsilon$. This situation is more unusual, and it does not occur in the example $W^{1, p}\left(S^{n}, S^{n}\right)$. It means that some map $f \in W^{1, p-\varepsilon}$ cannot be homotopied in $W^{1, p-\varepsilon}$ to any $g \in W^{1, p+\varepsilon}$. In particular, such a map $f$ cannot be smooth and it cannot be homotopied in $W^{1, p-\varepsilon}$ to any smooth map, so it must have a rather "robust" singularity!

We may now reformulate Theorem 15 and Theorem 19 using the above notion.

Theorem 21. We have $C T\left(S^{2}, S^{2}\right)=\{2\}$ or more generally $C T\left(S^{n}, S^{n}\right)=\{n\}$ for any $n \geq 2$.

Theorem 22. We have $C T\left(S^{n} \times \Lambda, S^{n}\right)=\{n+1\}$, where $\Lambda$ is the unit ball in $\mathbb{R}^{k}$ (for any $k \geq 1$ ).

We also observed in H. Brezis and Y. Li [19] that a change in topology may occur for several values of $p$, not just one, as in the above examples. Here is such a situation, with a "cascade of mergings".

Theorem 23. We have $C T\left(S^{1} \times S^{2}, S^{1} \times S^{2}\right)=\{2,3\}$.

This is somewhat natural because we have here (at least) two invariants: write $f=\left(f_{1}(x, y), f_{2}(x, y)\right)$ and set $d_{1}=\operatorname{deg} f_{1}(\cdot, y)$ and $d_{2}=\operatorname{deg} f_{2}(x, \cdot)$. Note that $d_{1}$ is well-defined and independent of $y \in S^{2}$ when $f \in W^{1, p}$ and $p \geq 2$ (by Theorem 18), while $d_{2}$ is well-defined and independent of $x \in S^{1}$ only when $p \geq 3$ (again by Theorem 18). Some new invariants appear, as $p$ increases from 1 to $\infty$, when crossing the values $p=2$ and $p=3$.

We also proposed in H. Brezis and Y. Li [19] two conjectures: 
Conjecture 1. $C T(M, N)$ consists only of integers; i.e., change of topology for $W^{1, p}(M, N)$ occurs only when $p$ is an integer.

Conjecture 2. Any map $f \in W^{1, p}(M, N)$ can always be connected in $W^{1, p}(M, N)$ to a smooth map (for any $p$ and for any $M, N$ ).

Both conjectures were solved in a beautiful piece of work by F. B. Hang and F. H. Lin [33].

The answer to Conjecture 1 is positive:

Theorem 24 (F. B. Hang and F. H. Lin [33]). $C T(M, N)$ consists only of integers.

Theorem 24 is an immediate consequence of the following remarkable result which provides a "reduction" of the study of homotopy classes for $W^{1, p}$ to more classical concepts in Topology. Given $p \geq 2$, let $M^{[p]-1}$ be the ([p]-1)-skeleton of $M$.

Theorem 25 (F. B. Hang and F. H. Lin [33]). Let $f, g \in W^{1, p}(M, N)$. Then $f \sim g$ in $W^{1, p}$ if and only if $f_{\mid \Sigma} \sim g_{\mid \Sigma}$ in $C^{0}$ for every generic $\Sigma$ in $M^{[p]-1}$.

Note that $\operatorname{dim} \Sigma \leq[p]-1$, and for a generic $\Sigma, f_{\mid \Sigma} \in W^{1, p}(\Sigma)$, while $p>\operatorname{dim} \Sigma$ (since $[p] \geq \operatorname{dim} \Sigma+1$ ). Thus for a generic $\Sigma, f_{\mid \Sigma} \in C^{0}$, by the Sobolev embedding.

Concerning Conjecture 2, we had presented in H. Brezis and Y. Li [19] several cases where Conjecture 2 is true: for example when $\operatorname{dim} M=2$ (for any $N$ ), or $\operatorname{dim} M=3$ and $\partial M \neq \phi$ (for any $N$ ), or $N=S^{1}$ (for any $M$ ). However, F. B. Hang and F. H. Lin [33] found some situations where Conjecture 2 fails. Here is such an example:

Theorem 26 (F. B. Hang and F. H. Lin [33). Let $M=\mathbb{R P}^{3}$ and $N=\mathbb{R P}^{2}$. Then $C T(M, N)=\{2,3\}$. Moreover there are maps $f \in W^{1, p}(M, N)$, with $p \in(2,3)$, such that $f$ cannot be connected in $W^{1, p}$ to any smooth map.

Open Direction 9. Compute $C T(M, N)$ when $\mathrm{M}$ and $\mathrm{N}$ are simple manifolds, e.g. spheres, products of spheres, product of spheres with balls, etc.

Acknowledgments. The author is partially sponsored by an EC Grant through the RTN Program "Fronts-Singularities", HPRN-CT-2002-00274. He is also a member of the Institut Universitaire de France.

\section{REFERENCES}

1. G. Alberti, S. Baldo and G. Orlandi, Functions with prescribed singularities (to appear).

2. F. Almgren and E. Lieb, Singularities of energy minimizing maps from the ball to the sphere: Examples, counterexamples, and bounds, Ann.of Math. 128 (1988), 483-530. MR 91a:58049

3. Th. Aubin, Equations différentielles nonlinéaires et problème de Yamabe concernant la courbure scalaire, J. Math. Pures et Appl. 55 (1976), 269-296. MR 55:4288

4. F. Bethuel, The approximation problem for Sobolev maps between two manifolds, Acta Math. 167 (1991), 153-206. MR 92f:58023

5. A characterization of maps in $H^{1}\left(B^{3}, S^{2}\right)$ which can be approximated by smooth maps, Ann. Inst. H. Poincaré, Anal. Non Linéaire 7 (1990), 269-286. MR 91f:58013

6. - On the singular set of stationary harmonic maps, Manuscripta Math. 78 (1993), 417-443. MR 94a:58047]

7. F. Bethuel, H. Brezis and J.-M. Coron, Relaxed energies for harmonic maps, in "Variational Methods" (H. Berestycki, J.-M. Coron and I. Ekeland, eds.), Birkhäuser, 1990. MR 94a:58046

8. F. Bethuel, H. Brezis and F. Hélein, Ginzburg-Landau Vortices, Birkhäuser, 1994. MR 95c:58044 
9. F. Bethuel and X. Zheng, Density of smooth functions between two manifolds in Sobolev spaces, J. Funct. Anal. 80 (1988), 60-75. MR 89i:58015

10. J. Bourgain, H. Brezis and P. Mironescu, Lifting in Sobolev spaces, J. Analyse Math. 80 (2000), p. 37-86. MR 2001h:46044

11. Another look at Sobolev spaces, Optimal Control and Partial Differential Equations (J. L. Menaldi, E. Rofman and A. Sulem, eds.), a volume in honor of A. Bensoussan's 60th birthday, IOS Press, 2001, pp. 439-455.

12. $-H^{1 / 2}$ maps with values into the circle: Minimal connections, lifting and the GinzburgLandau equation (to appear). Part of the results were announced in a note by the same authors: On the structure of the Sobolev space $H^{1 / 2}$ with values into the circle, C. R. Acad. Sci. Paris Ser. I Math. 331 (2000), 119-124. MR 2001m:46068

13. H. Brezis, $S^{k}$-valued maps with singularities, Topics in the Calculus of Variations (M. Giaquinta, ed.), Lecture Notes in Math., vol. 1365, Springer, 1989, 1-30. MR 90f:58029

14. - How to recognize constant functions. Connections with Sobolev spaces, Russian Math. Surveys, volume in honor of M. Vishik (to appear).

15. H. Brezis and F. Browder, Partial differential equations in the 20th century, Advances in Math. 135 (1998), 76-144, and Enciclopedia Italiana (to appear). MR 99i:35001

16. H. Brezis and J.-M. Coron, Large solutions for harmonic maps in two dimensions, Comm. Math. Phys. 92 (1983), 203-215. MR 85a:58022

17. Multiple solutions of H-systems and Rellich's conjecture, Comm. Pure Appl. Math. 37 (1984), 149-187. MR 85i:53010

18. H. Brezis, J.-M. Coron and E. Lieb, Harmonic maps with defects, Comm. Math. Phys. 107 (1986), 649-705. MR 88e:58023

19. H. Brezis and Y. Li, Topology and Sobolev spaces, J. Funct. Anal. 183 (2001), 321-369. MR 2002h:58009

20. H. Brezis, Y. Li, P. Mironescu and L. Nirenberg, Degree and Sobolev spaces, Topological Methods in Nonlinear Analysis 13 (1999), 181-190. MR 2001a:47065

21. H. Brezis and L. Nirenberg, Degree theory and BMO, Part I : compact manifolds without boundaries, Selecta Math. 1 (1995), 197-263. MR 96g:58023

22. J. Eells and L. Lemaire, A report on harmonic maps, Bull. London Math. Soc. 10 (1978), 1-68. MR 82b:58033

23. _ Another report on harmonic maps, Bull. London Math. Soc. 20 (1988), 385-524. MR 89i:58027

24. J. Ericksen, Equilibrium of liquid crystals, in "Advances in Liquid Crystals 2" (G. Brown, ed.), Acad. Press, 1976, 233-299.

25. J. Ericksen and D. Kinderlehrer (editors), Theory and applications of liquid crystals, IMA Series, Vol. 5, Springer, 1987. MR 88d:82007

26. L. C. Evans, Partial regularity for stationary harmonic maps into spheres, Arch. Rational Mech. Anal. 116 (1991), 101-113. MR 93m:58026

27. H. Federer, Geometric measure theory, Springer, 1969. MR 41:1976

28. M. Giaquinta, Multiple integrals in the calculus of variations and non-linear elliptic systems, Princeton Univ. Press, 1983. MR 86b:49003

29. M. Giaquinta and S. Hildebrandt, A priori estimates for harmonic mappings, J. Reine Angew. Math. 336 (1982), 124-164. MR 84b:58035

30. M. Giaquinta, G. Modica and J. Souček, Cartesian Currents in the Calculus of Variations, Springer, 1998. MR 2000b:49001a MR 2000b:49001b

31. E. Giusti (editor), Harmonic mappings and minimal immersions, Lecture Notes in Math. Vol. 1161, Springer, 1985; includes lectures by S. Hildebrandt, J. Jost and L. Simon. MR 86j:58037

32. R. Hamilton, Harmonic maps of manifolds with boundary, Lecture Notes in Math. 471, Springer, 1975. MR 58:2872

33. F. B. Hang and F. H. Lin, Topology of Sobolev mappings (to appear).

34. R. Hardt, D. Kinderlehrer and F. H. Lin, Existence and partial regularity of static liquid crystal configurations, Comm. Math. Phys. 105 (1986), 547-570. MR 88a:35207

35. R. Hardt and F. H. Lin, A remark on $H^{1}$ mappings, Manuscripta Math. 56 (1986), 1-10. MR 87k:58068

36. Singularities of p-energy minimizing unit vector-fields on planar domains, Calc. of Variations and PDE 3 (1995), 311-342. MR 97d:58060 
37. F. Hélein, Régularité des applications faiblement harmoniques entre une surface et une variété riemannienne, C. R. Acad. Sci. Paris 312 (1991), 591-596. MR 92e:58055

38. - Harmonic maps, conservation laws and moving frames, 2nd ed., Cambridge Univ. Press, 2002.

39. D. Hilbert, Uber das Dirichletsche Prinzip, Jahresbericht Deut. Math.-Ver. VIII, 1900, 184188 (also in J. Reine Angew. Math. 129 (1905), 63-67) and Math. Ann 59 (1904), 161-184.

40. S. Hildebrandt, Nonlinear elliptic systems and harmonic mappings, Proc. 1980 Beijing Symp. Diff. Geom. Diff. Eq., Science Press, Beijing, 1982, 481-615. MR 85k:35078

41. R. L. Jerrard and H. M. Soner, Functions of bounded higher variation (to appear).

42. J. Jost, Harmonic mappings between surfaces, Lecture Notes in Math., vol. 1062, Springer, 1984.

43. sphere with non-constant boundary values, J. Diff. Geom. 19 (1984), 393-401. MR 86b:58031

44. S. Klainerman and M. Machedon, Space-time estimates for null forms and the local existence theorem, Comm. Pure Appl. Math. 46 (1993), 1221-1268. MR 94h:35137

45. - Smoothing estimates for null forms and applications, Duke Math. J. 81 (1995), 99133. MR 97h:35022

46. M. Kléman, Points, lines and walls, John-Wiley, 1983. MR 85e:82058

47. F. H. Lin and T. Rivière, Complex Ginzburg-Landau equations in high dimensions and codimension-two area-minimizing currents, J. Eur. Math. Soc. 1 (1999), 237-311; Erratum 2 (2000), 87-91. MR 2000g:49048 MR 2001a:49041

48. C. Morrey, Multiple Integrals in the Calculus of Variations, Springer, 1966. MR 34:2380

49. H. Poincaré, Sur les équations aux dérivées partielles de la physique mathématique, Amer. J. Math. 12 (1980), 211-294

50. — Théorie du potential newtonien, Carré et Naud, 1899; reprinted J. Gabay, 1990.

51. T. Rivière, Everywhere discontinuous harmonic maps into spheres, Acta Math. 175 (1995), 197-226. MR 96k:58059

52. _ Line vortices in the U(1) - Higgs model, Control, Opt. and Calc. of Variations 1 (1996), 77-167. MR 97g:58043

53. J. Rubinstein and P. Sternberg, Homotopy classification of minimizers of the GinzburgLandau energy and the existence of permanent currents, Comm. Math. Phys. 179 (1996), 257-263. MR 97f:35208

54. J. Sacks and K. Uhlenbeck, The existence of minimal immersions of 2-spheres, Ann. of Math. 113 (1981), 1-24. MR 82f:58035

55. R. Schoen and K. Uhlenbeck, A regularity theory for harmonic maps, J. Diff. Geom. 17 (1982), 307-335. MR 84b:58037a correction in J. Diff. Geom. 18 (1983), 329. MR 84b:58037b

56. $\ldots$, Boundary regularity and the Dirichlet problem for harmonic maps, J. Diff. Geom. 18 (1983), 253-268. MR 85b:58037

57. _ Regularity of minimizing harmonic maps into the sphere, Invent. Math. 78 (1984), 89-100. MR 86a:58024

58. R. Schoen and S. T. Yau, Lectures on Harmonic Maps, International Press, 1997. MR 98i:58072

59. H. Wente, An existence theorem for surfaces of constant mean curvature, J. Math. Annal. 26 (1969), 318-344. MR 39:4788

60. H. Weyl, The method of orthogonal projection in potential theory, Duke Math. J. 7 (1940), 411-440.

61. B. White, Homotopy classes in Sobolev spaces and the existence of energy minimizing maps, Acta Math. 160 (1988), 1-17. MR 89a:58031

Department of Mathematics, Rutgers University, Piscataway, NJ 08854

E-mail address: brezis@math.rutgers.edu

Laboratoire Jacques-Louis Lions, Université Pierre et Marie Curie, Boîte courrier 187, 4 Place Jussieu, 75252 Paris Cedex 05, France

E-mail address: brezis@ann.jussieu.fr, brezis@ccr.jussieu.fr 